\title{
Diagnostic value of transcranial sonography for patients with Parkinson's disease in the chinese population: A meta and meta-regression analysis
}

\author{
Bo Liu, Wenjing Luo, Yingmin Mo, Chunying Wei and Min Han* \\ The Cadre ward in Department of Neurology, the people's hospital of guangxi zhuang autonomous region, No.6, Tao Yuan Road, Nanning, Guangxi, 530021, \\ China
}

\begin{abstract}
Background: To evaluate the diagnostic accuracy of substantia nigra hyper-echogenicity by transcranial sonography (TCS) for the diagnosis of Parkinson's disease (PD) in the Chinese population.

Methods: We searched the electronic databases of Cochrane Central Register of Controlled Trials (CENTRAL), Pubmed, Embase, China National Knowledge Infrastructure (CNKI), Chinese Biomedical Database (CBM) up to Dec 2018 for related articles. All the published population-based studies investigating diagnostic value of TCS for PD patients in China were searched and evaluated. A meta and meta-regression analysis was performed to calculate the available data from each research. Meta-Disc 1.4 statistics software was used.

Results: 23 studies with 1478 PD patients were analyzed. The pooled sensitivity, specificity, positive likelihood radio (LR+), negative 1ikellhood ratio (LR-) and diagnostic odds ratio (DOR) of TCS were 0.78 (95\% confidence interval (CI): 0.75, 0.80), 0.87 (95\% CI: 0.85, 0.89), 6.74 (95\% CI: 5.04, 8.31), 0.23 (95\% CI: 0.19, 0.28 ) and 31.32 (95\% CI: 21.28, 46.09), respectively, for differentiating PD patients from healthy controls. The AUC and Q index was 0.9119 and 0.8442 , respectively. In the subgroup and meta-regression analysis, we found that the important cause of heterogeneity was the sample size of $\mathrm{PD}$ patients $(\mathrm{P}=0.0051)$.
\end{abstract}

Conclusion: TCS is an effective and useful tool to detect and distinguish PD from healthy controls in the Chinese individuals.

\section{Introduction}

Parkinson's disease (PD) is the second most prevalent neurodegenerative disease in the world and is associated with the progressive loss of dopaminergic neurons in the substantia nigra (SN) [1]. Diagnosis of PD is usually based on clinical criteria [2]. A recent clinical pathology study demonstrated that in the early stage of the disease, only $50 \%$ PD patients received an accurate diagnosis of $\mathrm{PD}$ [3]. Besides, in specialized movement disorder centers, PD is wrongly diagnosed in 6 to $25 \%$ of cases [4]. The most diagnostic confusing diseases are atypical parkinsonian disorders (APD), including progressive supranuclear palsy (PSP), multiple system atrophy (MSA), corticobasal ganglionic degeneration (CBD) and dementia with Lewy bodies (DLB) [5-7]. Therefore, to improve the accuracy of the clinical diagnosis, there is a need to have a reliable and practical reference standard. Recently, several different diagnostic tools have been evaluated in their ability to detect nigrostriatal cell loss. TCS is one of the most widely used tests, which is a convenient and noninvasive diagnostic technique that allows imaging of the brain parenchyma in twodimensional black and white slices [8]. Moreover, some articles had reported substantia nigra hyperechogenicity in PD and assessed the diagnostic accuracy of TCS $[9,10]$. But, most of evidences came from European and American. Until now, there were only a few evidences on Chinese individuals, and the conclusions were discrepant $[11,12]$. Consequently, we conducted a meta-analysis to estimate the clinical utility of TCS in the Chinese PD individuals.

\section{Materials and methods}

\section{Literature search}

Eligible studies were identified by searching in the Cochrane Central Register of Controlled Trials (CENTRAL), Pubmed, Embase, China National Knowledge Infrastructure (CNKI), Chinese Biomedical Database (CBM) for relevant trials without language restrictions using the following mesh search terms: "transcranial sonography" or "TCS", "Parkinson's disease" or "PD”. Terms were exploded whenever possible in each database. We fixed Dec. 2018 as the cut-off date for inclusion of studies.

\section{Inclusion criteria for relevant studies}

Studies were included if they met all of the following criteria: (1) Studies needed to assessed the ability of the TCS. (2) Diagnostic differentiation between PD and controls, the diagnosis of PD was made according to the United Kingdom Parkinson's Disease Society Brain

*Correspondence to: Min Han, the Cadre ward in Department of Neurology, the people's hospital of guangxi zhuang autonomous region, No.6, Tao Yuan Road, Nanning, Guangxi, 530021, China, E-mail: bliu881215@163.com

Key words: transcranial sonography, Parkinson's disease, Chinese population, meta-analysis

Received: October 29, 2019; Accepted: November 12, 2019; Published: November 15, 2019 
Bank Criteria [13] or the Movement Disorder and Parkinson's Disease Group of the Chinese Medical Association Branch of Neurology [14], (3) Original articles provided the extractable data on the diagnostic value of TCS in PD patients. (4) Clinical data regarding the publication year, ages, gender, sample size and diagnosis criteria was collected. (5) Studies would be excluded if the above information could not be obtained.

\section{Outcome measures for this review}

Two independent reviewers obtained full manuscripts of all citations that were likely to meet the predefined selection criteria. They independently reviewed the articles and extracted the data from the included studies. Areas of disagreement or uncertainty were resolved by discussion and consultation with a third reviewer. When multiple articles were published from a single study, we selected the report that contained the most complete and relevant data on the diagnostic value of TCS in PD patients. Two reviewers independently extracted the following data elements from each selected article: the study characteristics, number of PD patients and controls, and data regarding diagnostic value of TCS. The pooled sensitivity, specificity, LR+, LR- and DOR with 95\% CI were calculated to estimate the diagnostic value of TCS in PD patients. We calculated statistical heterogeneity by an $\mathrm{X}^{2}$ test on N-1 degrees of freedom with $\mathrm{P}<0.1$ indicating significant heterogeneity [15]. To evaluate the heterogeneity, we also used the $\mathrm{I}^{2}$ test, taking values in the range $0-100 \%$ [16]. $I^{2}$ values of $25 \%$ may be represent low, $50 \%$ modest, and $75 \%$ large heterogeneity, respectively [17]. When $\mathrm{P} \geq 0.1$ or $\mathrm{I}^{2} \leq 50 \%$ indicated a lack of heterogeneity, the fixed effect model was employed. Otherwise, the random effect model was chosen. Potential publication bias was examined using funnel plots [18]. A sensitivity analysis was also performed by repeating the meta-analysis and omitting each study at each iteration [19]. Potential publication bias was examined using funnel plots [20]. The calculations were performed using the Meta-Disc 1.4 statistical software.

\section{Results}

169 studies were identified from the initial search, we excluded 146 studies for the following reasons: reviews or case reports, the publications dealt with other topics, duplication of data, or not available as full text articles. Finally, we identified 23studies represented data from 1478 PD patients for the meta-analysis [1,11,12,21-39]. In 14 articles, PD patients were diagnosed according to the United Kingdom Parkinson's Disease Society Brain Bank Criteria (UK). In 9 articles, PD patients were diagnosed according to the Movement Disorder and Parkinson's Disease Group of the Chinese Medical Association Branch of Neurology (Chinese diagnosis criteria) [14]. The main characteristics of all included studies were summarized in Table 1. There was significant statistical heterogeneity among the studies, thus, a random effect model was employed. The pooled sensitivity of TCS for the diagnosis PD was $78 \%$ (95\% CI: $0.75,0.80$ ) (Figure 1) and the pooled specificity was $87 \%$ (95\% CI: 0.85, 0.89) (Figure 2). The pooled LR+, LR- and DOR of TCS were 6.74 (95\% CI: 5.04, 8.31) (Figure 3), 0.23 (95\% CI: 0.19, 0.28) (Figure 4) and 31.32 (95\% CI: 21.28, 46.09) (Figure 5), respectively. The ROC plane did not revealed no heterogeneity secondary to the threshold effect (Figure 6). In addition, the AUC and Q index showed in the symmetric SROC curve was 0.9119 and 0.8442 , respectively, which reflected the overall high level of accuracy (Figure 7). The results were consistent when we performed a secondary analysis by repeating the meta-analysis and omitting each study at each iteration. Publication bias may be acceptably low because all the funnel plots on the diagnostic value of TCS in the Chinese PD patients for the included studies did not reveal obvious signs of publication bias (Figure 8).

Furthermore, we used five items to separate subgroup analysis: the publication year (2010-2015; 2016-2018), age ( $\leq 65,>65)$, sample size $(\leq 50 ;>50)$, diagnosis criteria of PD (UK; Chinese diagnosis criteria), and male ratio $(\leq 55 \%,>55 \%)$. The studies taking place in 4 years ago had higher sensitivity, specificity, LR+ and DOR than those in the last 4 years $(0.8 \mathrm{vs} .0 .74,0.87$ vs.0.86, $6.51 \mathrm{vs} .6 .18,33.18 \mathrm{vs} .25 .98$, respectively), but lower LR- $(0.22 \mathrm{vs} .0 .26)$. The sensitivity, specificity,

Table 1. Characteristics of studies included in the meta-analysis

\begin{tabular}{|c|c|c|c|c|c|c|c|c|c|}
\hline Author & Years & Diagnosis criteria & Number of PD & Male rido (\%) & Ages & TP & FP & FN & $\mathbf{T N}$ \\
\hline Feng AZ & 2012 & Chinese diagnosis criteria & 30 & 53.33 & $54.3 \pm 7.9$ & 25 & 3 & 5 & 27 \\
\hline Qiao YN & 2016 & Chinese diagnosis criteria & 89 & 52.8 & 61.1 & 81 & 5 & 8 & 79 \\
\hline Liu X & 2018 & Chinese diagnosis criteria & 38 & 47.37 & $69.37 \pm 9.86$ & 28 & 3 & 10 & 39 \\
\hline Xu HW & 2017 & Chinese diagnosis criteria & 51 & 52.9 & $69.51 \pm 3.49$ & 41 & 12 & 10 & 39 \\
\hline Liu X & 2013 & Chinese diagnosis criteria & 42 & 81.9 & $62 \pm 14$ & 39 & 3 & 3 & 47 \\
\hline Zhuang JJ & 2015 & Chinese diagnosis criteria & 36 & 58.33 & $62.63 \pm 7.57$ & 32 & 2 & 4 & 30 \\
\hline Hu HY & 2016 & Chinese diagnosis criteria & 100 & 57.27 & $61.7 \pm 5.7$ & 73 & 5 & 27 & 45 \\
\hline Wang XP & 2017 & Chinese diagnosis criteria & 51 & 55.97 & 68.31 & 41 & 13 & 10 & 37 \\
\hline Yang LJ & 2012 & Chinese diagnosis criteria & 53 & 56.6 & $69.3 \pm 7.3$ & 40 & 7 & 13 & 46 \\
\hline Zhu JW & 2018 & UK & 36 & 52.78 & $61.7 \pm 11.2$ & 29 & 5 & 7 & 36 \\
\hline Luo WF & 2012 & UK & 110 & 49.1 & 58.7 & 88 & 11 & 22 & 99 \\
\hline Sheng YJ & 2012 & UK & 78 & 48.71 & 62.2 & 66 & 5 & 12 & 55 \\
\hline Zhang YC & 2010 & UK & 80 & 56.5 & $60.7 \pm 11$ & 58 & 10 & 22 & 70 \\
\hline Zhang LL & 2018 & UK & 65 & 53.8 & $65.34 \pm 10.68$ & 42 & 6 & 23 & 47 \\
\hline Chen $\mathrm{J}$ & 2013 & UK & 30 & 60 & $47.3 \pm 5.18$ & 25 & 2 & 5 & 28 \\
\hline Liu XJ & 2018 & UK & 30 & 60 & $56.5 \pm 5.01$ & 25 & 1 & 5 & 27 \\
\hline Wang $\mathrm{R}$ & 2011 & UK & 34 & 61.76 & $64.11 \pm 10.25$ & 31 & 4 & 3 & 34 \\
\hline Cao CJ & 2017 & UK & 122 & 64.75 & $60.0 \pm 10.5$ & 91 & 3 & 31 & 37 \\
\hline $\mathrm{LiC}$ & 2013 & UK & 92 & 56.5 & 62.23 & 74 & 26 & 18 & 73 \\
\hline Zhou HY & 2016 & UK & 201 & 69.15 & 61.43 & 124 & 17 & 77 & 75 \\
\hline Dong ZF & 2017 & UK & 38 & 57.89 & $67.3 \pm 8.6$ & 31 & 3 & 7 & 23 \\
\hline Yang J & 2017 & UK & 30 & 57.14 & 65.4 & 24 & 2 & 6 & 28 \\
\hline Zhang ZH & 2015 & UK & 42 & $66 \%$ & $65.2 \pm 7.3$ & 38 & 9 & 4 & 39 \\
\hline
\end{tabular}




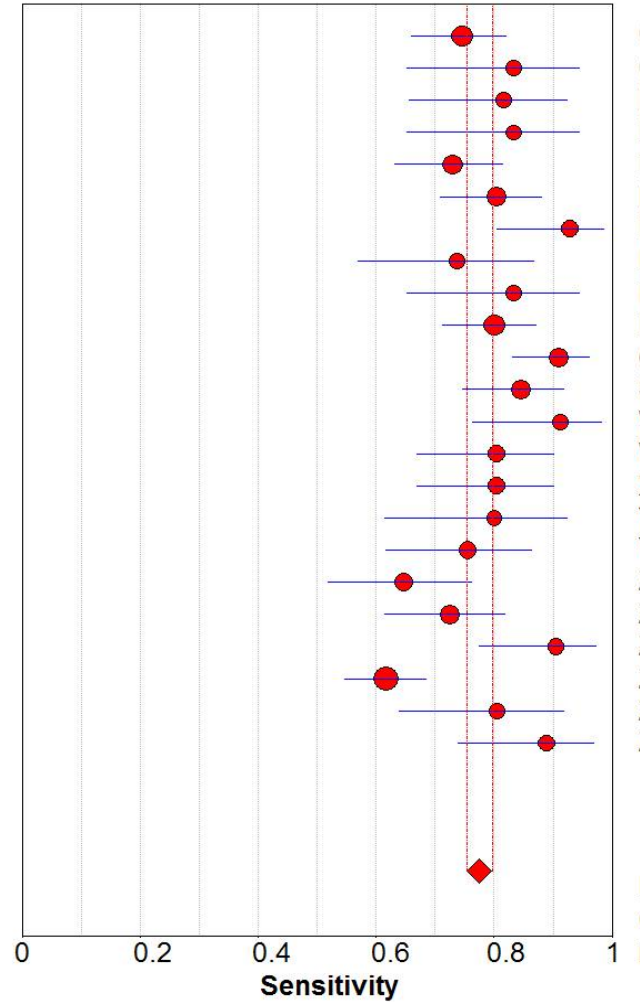

Cao CJ
Chen J
Dong ZF
Feng AZ
Hu HY
Li C
Liu X
Liu X
Liu XJ
Luo WF
Qiao YN
Sheng YJ
Wang R
Wang XP
Xu HW
Yang J
Yang LJ
Zhang LL
Zhang YC
Zhang ZH
Zhou HY
Zhu JW
Zhuang JJ

Sensitivity $(95 \% \mathrm{Cl})$

$0.75 \quad(0.66-0.82)$

$0.83 \quad(0.65-0.94)$

$0.82 \quad(0.66-0.92)$

$0.83 \quad(0.65-0.94)$

$0.73(0.63-0.81)$

$0.80 \quad(0.71-0.88)$

$0.93 \quad(0.81-0.99)$

$0.74 \quad(0.57-0.87)$

$0.83 \quad(0.65-0.94)$

$0.80(0.71-0.87)$

$0.91 \quad(0.83-0.96)$

$0.85 \quad(0.75-0.92)$

$0.91 \quad(0.76-0.98)$

$0.80 \quad(0.67-0.90)$

$0.80 \quad(0.67-0.90)$

$0.80 \quad(0.61-0.92)$

$0.75 \quad(0.62-0.86)$

$0.65 \quad(0.52-0.76)$

$0.73 \quad(0.61-0.82)$

$0.90 \quad(0.77-0.97)$

$0.62 \quad(0.55-0.68)$

$0.81 \quad(0.64-0.92)$

$0.89(0.74-0.97)$

Pooled Sensitivity $=0.78(0.75$ to 0.80$)$

Chi-square $=71.61 ; d f=22(p=0.0000)$

Inconsistency (I-square) $=69.3 \%$

Figure 1. The sensitivity of transcranial sonography for the diagnosis of Parkinson's disease in the Chinese population

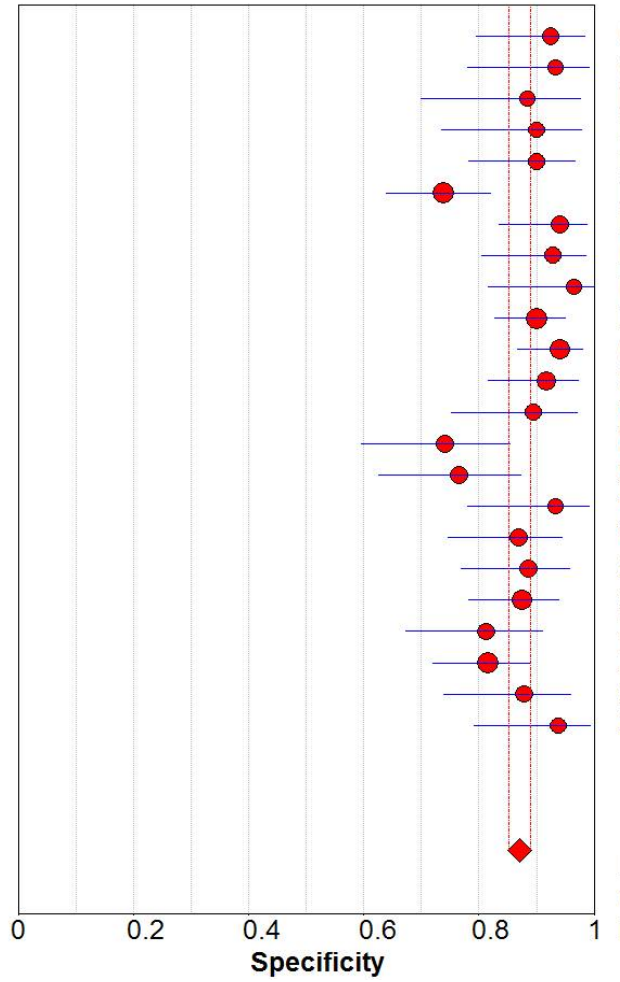

Cao CJ

Chen J

Dong ZF

Feng $A Z$

$\mathrm{Hu} \mathrm{HY}$

Li C

Liu $X$

Liu $X$

Liu XJ

Luo WF

Qiao YN

Sheng $Y J$

Wang $R$

Wang $X P$

Xu HW

Yang $\mathrm{J}$

Yang LJ

Zhang LL

Zhang YC

Zhang ZH

Zhou HY

Zhu JW

Zhuang JJ

Specificity $(95 \% \mathrm{Cl})$

$0.93 \quad(0.80-0.98)$

$0.93 \quad(0.78-0.99)$

$0.88 \quad(0.70-0.98)$

$0.90 \quad(0.73-0.98)$

$0.90 \quad(0.78-0.97)$

$0.74 \quad(0.64-0.82)$

$0.94 \quad(0.83-0.99)$

$0.93 \quad(0.81-0.99)$

$0.96 \quad(0.82-1.00)$

$0.90 \quad(0.83-0.95)$

$0.94 \quad(0.87-0.98)$

$0.92 \quad(0.82-0.97)$

$0.89 \quad(0.75-0.97)$

$0.74 \quad(0.60-0.85)$

$0.76 \quad(0.63-0.87)$

$0.93(0.78-0.99)$

$0.87 \quad(0.75-0.95)$

$0.89 \quad(0.77-0.96)$

$0.88 \quad(0.78-0.94)$

$0.81 \quad(0.67-0.91)$

$0.82 \quad(0.72-0.89)$

$0.88 \quad(0.74-0.96)$

$0.94 \quad(0.79-0.99)$

Pooled Specificity $=0.87(0.85$ to 0.89$)$

Chi-square $=46.37 ; \mathrm{df}=22(p=0.0018)$

Inconsistency (I-square) $=52.6 \%$

Figure 2. The specificity of transcranial sonography for the diagnosis of Parkinson's disease in the Chinese population 


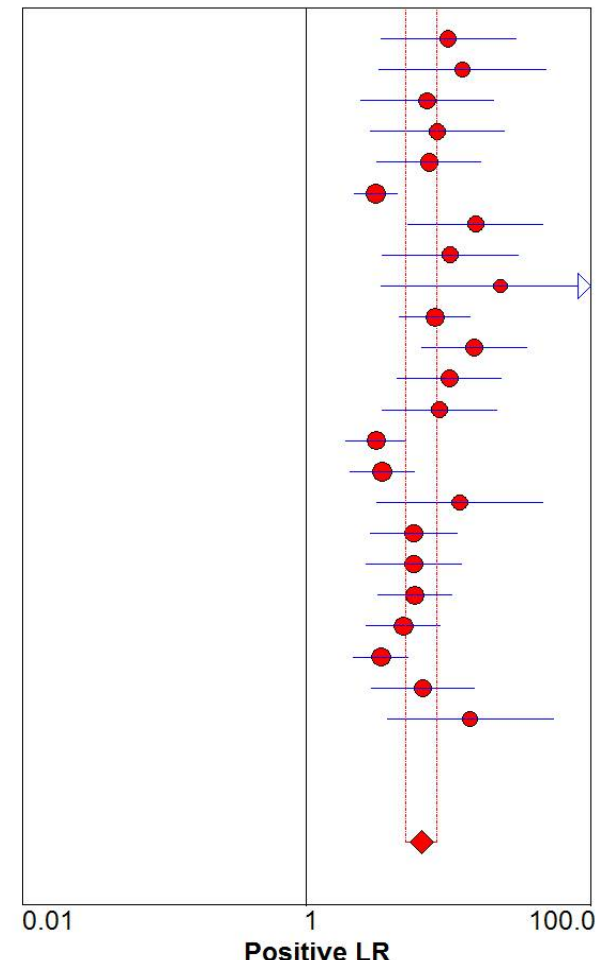

Cao CJ

Chen J

Dong ZF

Feng $A Z$

$\mathrm{Hu} \mathrm{HY}$

Li C

Liu $X$

Liu $X$

Liu XJ

Luo WF

Qiao YN

Sheng YJ

Wang $\mathrm{R}$

Wang XP

Xu HW

Yang $\mathrm{J}$

Yang LJ

Zhang LL

Zhang YC

Zhang $\mathrm{ZH}$

Zhou HY

Zhu JW

Zhuang JJ
Positive LR (95\% Cl)

$9.95 \quad(3.33-29.68)$

$12.50 \quad(3.25-48.14)$

$7.07 \quad(2.41-20.72)$

$8.33 \quad(2.81-24.67)$

$7.30 \quad(3.15-16.91)$

$3.06 \quad(2.17-4.33)$

$15.48 \quad(5.15-46.51)$

$10.32(3.41-31.20)$

$23.33(3.38-160.96)$

$8.00 \quad(4.53-14.12)$

$15.29(6.52-35.86)$

$10.15 \quad(4.36-23.63)$

$8.66 \quad(3.41-22.02)$

$3.09(1.90-5.03)$

$3.42(2.05-5.71)$

$12.00(3.11-46.33)$

$5.71 \quad(2.82-11.59)$

$5.71 \quad(2.63-12.39)$

$5.80 \quad(3.20-10.52)$

$4.83(2.66-8.77)$

$3.34(2.14-5.20)$

$6.61(2.86-15.25)$

$14.22(3.70-54.69)$

Random Effects Model

Pooled Positive LR = 6.47 (5.04 to 8.31)

Cochran-Q $=53.47 ; \mathrm{df}=22(\mathrm{p}=0.0002)$

Inconsistency (I-square) $=58.9 \%$

Tau-squared $=0.1903$

Figure 3. The positive likelihood radio of transcranial sonography for the diagnosis of Parkinson's disease in the Chinese population

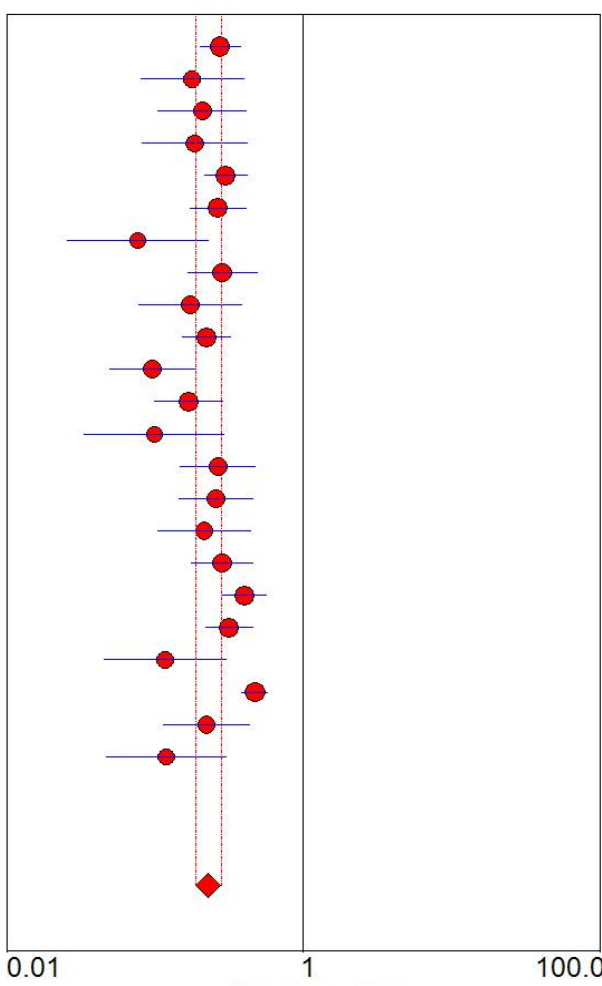

Negative LR
Cao CJ

Chen J

Dong ZF

Feng $A Z$

$\mathrm{Hu} \mathrm{HY}$

Li C

Liu $X$

Liu $X$

Liu XJ

Luo WF

Qiao YN

Sheng $Y J$

Wang $R$

Wang XP

Xu HW

Yang J

Yang LJ

Zhang LL

Zhang YC

Zhang $\mathrm{ZH}$

Zhou HY

Zhu JW

Zhuang JJ
Negative LR $(95 \% \mathrm{Cl}$

$0.27 \quad(0.20-0.38)$

$0.18 \quad(0.08-0.40)$

$0.21 \quad(0.11-0.41)$

$0.19 \quad(0.08-0.42)$

$0.30 \quad(0.21-0.42)$

$0.27 \quad(0.17-0.41)$

$0.08 \quad(0.03-0.23)$

$0.28 \quad(0.17-0.49)$

$0.17 \quad(0.08-0.39)$

$0.22 \quad(0.15-0.32)$

$0.10 \quad(0.05-0.19)$

$0.17 \quad(0.10-0.28)$

$0.10 \quad(0.03-0.29)$

$0.26 \quad(0.15-0.47)$

$0.26 \quad(0.14-0.46)$

$0.21 \quad(0.10-0.44)$

$0.28 \quad(0.17-0.46)$

$0.40 \quad(0.28-0.56)$

$0.31 \quad(0.22-0.45)$

$0.12 \quad(0.05-0.30)$

$0.47 \quad(0.38-0.57)$

$0.22 \quad(0.11-0.43)$

$0.12 \quad(0.05-0.30)$

Random Effects Model

Pooled Negative LR $=0.23$ (0.19 to 0.28$)$

Cochran $-\mathrm{Q}=77.44 ; \mathrm{df}=22(\mathrm{p}=0.0000)$

Inconsistency (I-square) $=71.6 \%$

Tau-squared $=0.1499$

Figure 4. The negative likellhood ratio of transcranial sonography for the diagnosis of Parkinson's disease in the Chinese population 


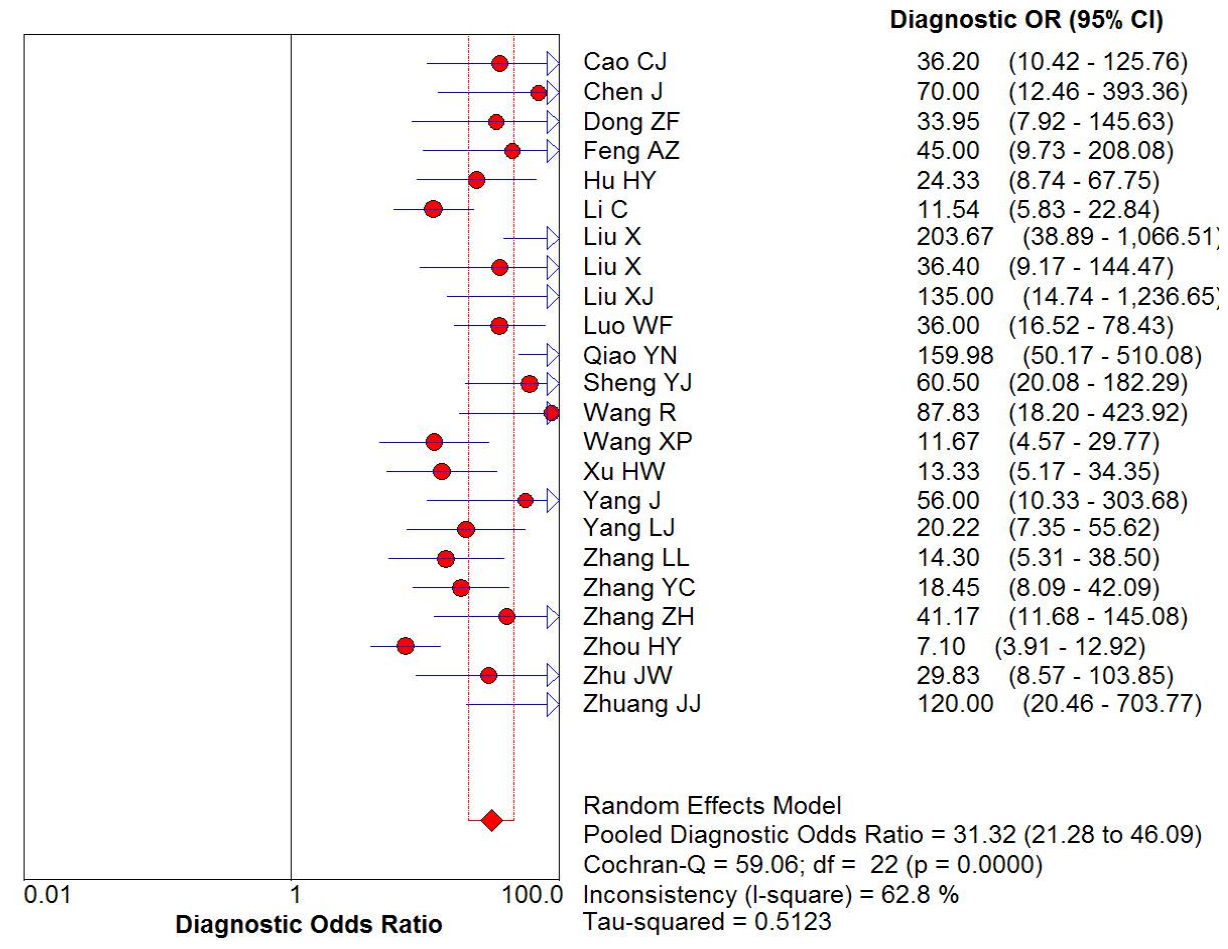

Figure 5. The diagnostic odds ratio of transcranial sonography for the diagnosis of Parkinson's disease in the Chinese population

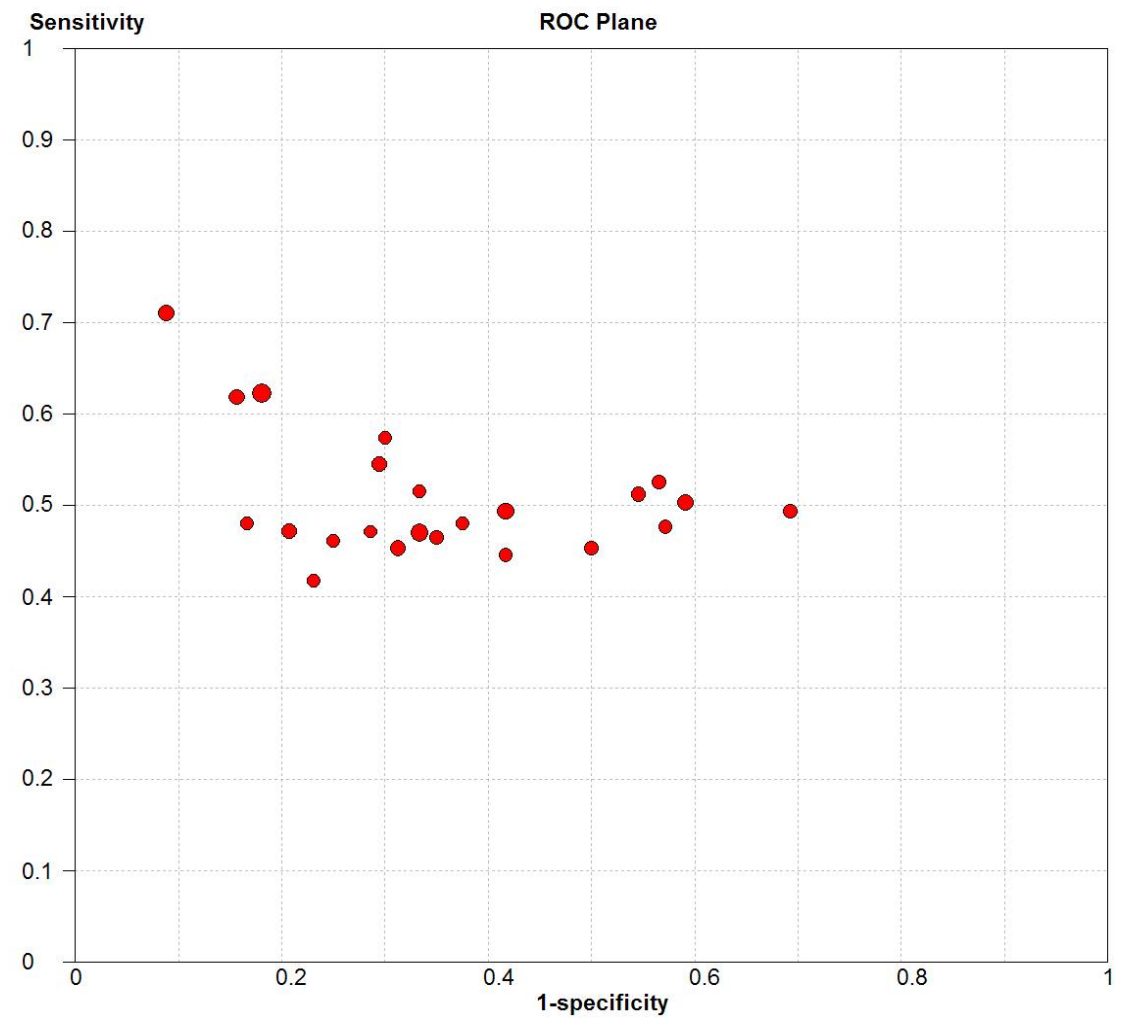

Figure 6. Sensitivity versus 1-specificity in ROC plane for each included study 
LR+, LR- and DOR for the studies with age $>65$ were $0.77,0.84$, $4.81,0.27,19.9$ respectively; while for those with age $\leq 65$, the results increased to 0.78 (sensitivity), 0.88 (specificity), 7.63 (LR+), 39.72 (DOR), but LR- reduced to 0.21 . The sensitivity, specificity, LR+ and

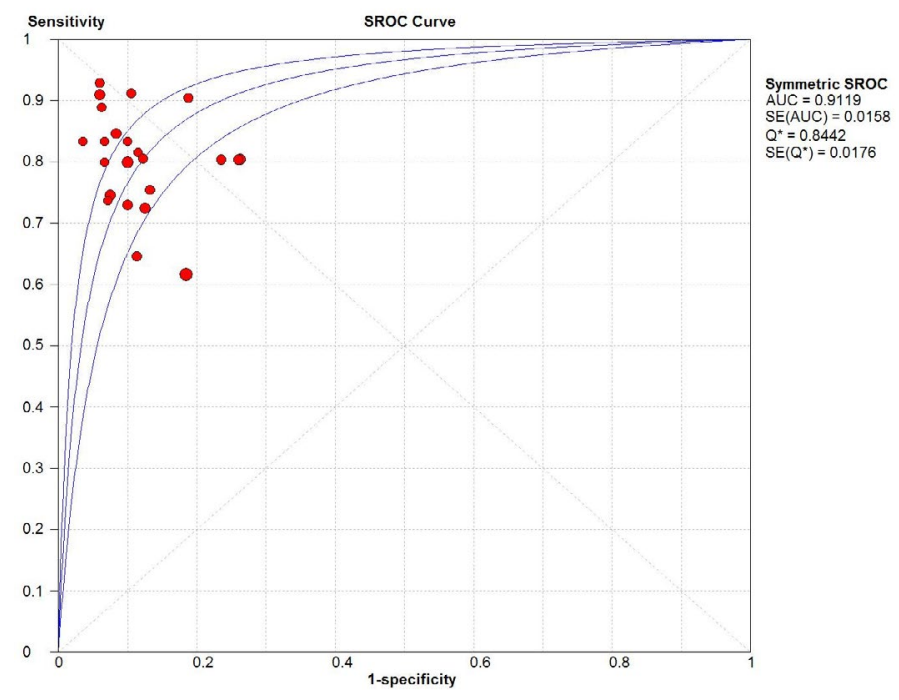

Figure 7. SROC of the accuracy of transcranial sonography for the diagnosis of Parkinson's disease in the Chinese population

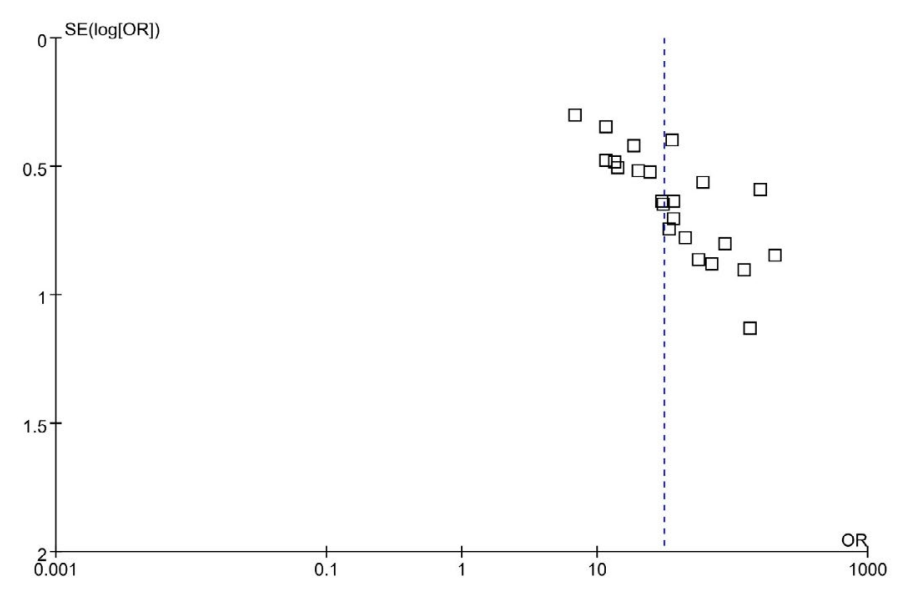

Figure 8. Funnel plots for the included studies
DOR of TCS for the studies with a sample size below 50 individuals was higher than those with a sample size over 50 (0.85vs.0.75, $0.91 \mathrm{vs} .0 .85$, 8.11 vs. $5.38,55.95$ vs. 21.33 , respectively), besides LR- (0.18vs.0.27). The sensitivity, specificity, LR+ and DOR of TCS for the subgroup with Chinese diagnosis criteria was higher than those with UK (0.82vs.0.76, $0.88 v s .0 .87,7.11$ vs. $6.17,37.55 \mathrm{vs} .27 .94$, respectively), except the LR(0.21vs.0.24). The last one, the sensitivity, specificity, LR+, LR- and DOR of TCS for the studies with more male PD patients was lower, (0.76vs. 0.79, 0.85vs.0.89, 6.13vs.6.96, 0.85vs.0.89, 31.73 vs. 31.90 , respectively). The results of the subgroup analysis were shown in Table 2. To determine the effect of these factors for the heterogeneity, we further conducted a meta-regression analysis with the covariates which included in subgroup analysis. Notwithstanding, when the metaregression analysis was performed, the sample size was the important cause for the heterogeneity $(\mathrm{P}=0.0051)$, Table 3 .

\section{Discussion}

Numerous ultrasound studies have suggested that a typical enlarged area of echogenicity in the substantia nigra can help diagnose PD in European and American [40,41]. However, it is still unknown the exact diagnostic accuracy of substantia nigra hyper-echogenicity by TCS for the diagnosis of PD in the Chinese population. Some studies $[23,26]$ found that PD patients had high incidences of SN hyperechogenicity detected by TCS, and TCS could improve the specificity of diagnosis for PD. However, Zhang YC, et al. [22] reported that TCS might provide some valuable information for clinical diagnosis of $\mathrm{PD}$, but some of the older individuals were unsuitable to the exam for their unadequated temporale bone windows. Until now, there is no consensus on whether TCS is a useful and accurate method in the Chinese population. So, further studies are required to identify the diagnostic value of TCS in detection of PD in the Chinese population. To our knowledge, the present study is the first meta-analysis determining diagnostic value of TCS in the diagnosis of PD in the Chinese population. Our study showed the pooled sensitivity and specificity of TCS were $78 \%$ and $87 \%$, respectively. The results of the current study are consistent with previous findings in European populations [42,43]. As we expected, in the subgroup of sample size, studies that used larger sample size $(>50)$ would report a lower sensitivity, specificity, LR+, and DOR, while a higher LR-. Moreover, the sample size was confirmed to be the significant cause of heterogeneity in the meta-regression analysis $(\mathrm{P}=0.0051)$. In our study, the age and male ratio subgroups showed the lower sensitivity, specificity, LR+, and DOR, while a higher LR-.

Table 2. Subgroup analysis of the diagnostic value of transcranial sonography in Parkinson's disease

\begin{tabular}{|c|c|c|c|c|c|c|}
\hline Subgroup & Number of studies & sensitivity $(95 \% \mathrm{CI})$ & specificity (95\% CI) & $\begin{array}{c}\text { Positive } \\
\text { LR }(95 \% \text { CI) }\end{array}$ & $\begin{array}{c}\text { Negative } \\
\text { LR }(95 \% \text { CI) }\end{array}$ & diagnostic OR $(95 \% \mathrm{CI})$ \\
\hline \multicolumn{7}{|c|}{ Publication year } \\
\hline $2010-2015$ & 11 & $0.80(0.77 ; 0.83)$ & $0.87(0.84 ; 0.89)$ & $6.51(4.54 ; 9.34)$ & $0.22(0.15 ; 0.31)$ & $33.18(18.18 ; 60.58)$ \\
\hline $2016-2018$ & 12 & $0.74(0.71 ; 0.77)$ & $0.87(0.84 ; 0.90)$ & $6.18(4.28 ; 8.92)$ & $0.26(0.20 ; 0.34)$ & $25.98(14.75 ; 45.76)$ \\
\hline \multicolumn{7}{|l|}{ Sample size } \\
\hline$\leq 50$ & 11 & $0.85(0.81 ; 0.88)$ & $0.91(0.87 ; 0.93)$ & $8.11(5.97 ; 11.02)$ & $0.18(0.15 ; 0.23)$ & $55.95(35.23 ; 88.85)$ \\
\hline$>50$ & 12 & $0.75(0.72 ; 0.78)$ & $0.85(0.83 ; 0.88)$ & $5.38(3.95 ; 7.33)$ & $0.27(0.22 ; 0.34)$ & $21,33(13.37 ; 34.37)$ \\
\hline \multicolumn{7}{|c|}{ Diagnosis criteria of PD } \\
\hline UK & 14 & $0.76(0.73 ; 0.78)$ & $0.87(0.84 ; 0.89)$ & $6.17(4.58 ; 8.31)$ & $0.24(0.19 ; 0.31)$ & $27.94(17.49 ; 44.64)$ \\
\hline Chinese diagnosis criteria & 9 & $0.82(0.78 ; 0.85)$ & $0.88(0.85 ; 0.91)$ & $7.11(4.41 ; 11.47)$ & $0.21(0.15 ; 0.29)$ & $37.55(18.89 ; 74.66)$ \\
\hline \multicolumn{7}{|c|}{ Age } \\
\hline$\leq 65$ & 15 & $0.78(0.75 ; 0.80)$ & $0.88(0.86 ; 0.90)$ & $7.63(5.37 ; 10.84)$ & $0.21(0.16 ; 0.28)$ & $39.72(22.82 ; 69.13)$ \\
\hline$>65$ & 8 & $0.77(0.73 ; 0.82)$ & $0.84(0.80 ; 0.88)$ & $4.81(3.55 ; 6.57)$ & $0.27(0.22 ; 0.34)$ & $19.94(13.36 ; 29.72)$ \\
\hline \multicolumn{7}{|l|}{ Male ratio } \\
\hline$\leq 55$ & 9 & $0.79(0.76 ; 0.83)$ & $0.89(0.86 ; 0.92)$ & $6.96(5.03 ; 9.63)$ & $0.23(0.18 ; 0.31)$ & $31.90(19.20 ; 53.01)$ \\
\hline$>55$ & 14 & $0.76(0.73 ; 0.79)$ & $0.85(0.83 ; 0.88)$ & $6.13(4.36 ; 8.62)$ & $0.22(0.17 ; 0.37)$ & $31.73(18.27 ; 55.18)$ \\
\hline
\end{tabular}


Table 3. Meta-regression analysis of potential moderators to explain heterogeneity of diagnostic value of transcranial sonography in PD

\begin{tabular}{|c|c|c|c|c|}
\hline & Meta-regression coefficient & SE & P & R5\% CI \\
\hline Publication year & -0.329 & 0.3673 & 0.3838 & 0.72 \\
\hline Sample size & -1.121 & 0.3459 & 0.0051 & $0.33 ; 1.57$ \\
\hline Diagnosis criteria of PD & -0.554 & 0.3481 & 0.1313 & $0.16 ; 0.68$ \\
\hline Age & -0.561 & 0.3760 & 0.1548 & 0.57 \\
\hline Male ratio & -0.258 & 0.3306 & 0.4473 & 0.57 \\
\hline
\end{tabular}

The results indicated gender and age were the factors relating higher incidences of $\mathrm{SN}$ hyperechogenicity, which were consistent with previous study[26]. However, Zhuang JJ, etal. reported that the area of bilateral substantia nigra echo was negatively correlated with gender, but positively correlated with age [30]. Additionally, Zhou HY, et al. thought that males had larger SNL echogenic areas compared to females, while age had no effect on the SN echogenic areas [1]. Thus, further studies are required to address this issue. In the subgroup of diagnosis criteria of $\mathrm{PD}$, the result is also significantly different. The sensitivity, specificity, LR+ and DOR of TCS for the subgroup with Chinese diagnosis criteria were higher than those with UK, except LR-. But in the further meta-regression analysis, the diagnosis criteria were not the cause of heterogeneity $(\mathrm{P}=0.1313)$. Therefore, we could use both $\mathrm{UK}$ and Chinese diagnosis criteria to diagnose PD patients in our daily work.

Although SN hyperechogenicity is a common feature of PD patients, the quality of the temporal bone window might cause the difference for the diagnosis by using TCS. And 10-20\% European were excluded from TCS studies due to this issue [44]. In China, TCS examination failed in $8.08-12.08 \%$ of the individuals [22], which was much lower than previous reports. we speculated that the differences might be explained by the racial differences in the temporal skull thickness and the size of brain between European and Asian [45]. In addition, some included studies found that SN echogenicity significantly correlated with UPDRS part II scores in the Chinese PD patients [26,30], suggesting that increased SN echogenicity might reflect more severe disease disability or poorer response to medication. However, due to the relatively low penetration rate and relatively low sensitivity of TCS, there is a need to demonstrate these findings in the future studies.

Overall the evidence for the use of TCS was strong; our study also had several limitations. First, although our study had a large sample size compared to most of the previous studies, the sample size in some of the included studies was small. We have found that sample size was the important cause for the actual prevalence of heterogeneity in our meta-regression analysis. Second, the heterogeneity among the studies was significant. Although we attempted to find the causes of it by conducting subgroup analysis and meta-regression analysis, the heterogeneity among studies persisted. Finally, almost all these studies were retrospective and involved patients with well-established diagnoses and long-disease duration. Therefore, prospective cohort study for diagnostic accuracy would be practically difficult but important for future research.

\section{Conclusion}

In summary, our present study demonstrated that SN hyperechognicity could help to discriminate PD from healthy controls; TCS was a useful and accurate method in the diagnosis of PD in the Chinese population. However, TCS had its own limitations, which included its dependency on the bone window and operator experience.

\section{Acknowledgments}

We would like to acknowledge the investigators for their helpful comments on this paper.

\section{Conflicts of interest}

We have no conflicts of interest regarding the content of this article.

\section{References}

1. Zhou HY, Sun Q, Tan YY (2016) Substantia nigra echogenicity correlated with clinical features of parkinson's disease. Parkinsonism Relat Disord 24: 28-33.

2. Mehnert S (2010) Transcranial sonography for diagnosis of Parkinson's disease. BMC Neurol 10: 9.

3. Adler CH (2014) Low clinical diagnostic accuracy of early vs advanced Parkinson disease: clinicopathologic study. Neurology 83: 406-412.

4. Hughes AJ (2002) The accuracy of diagnosis of parkinsonian syndromes in a specialist movement disorder service. Brain 125: 861-70.

5. Zhou HY (2018) The role of substantia nigra sonography in the differentiation of Parkinson's disease and multiple system atrophy. Transl Neurodegener 5: 7.

6. Bouwmans AEP (2013) Specificity and sensitivity of transcranial sonography of the substantia nigra in the diagnosis of Parkinson's disease: prospective cohort study in 196 patients. BMJ Open 3: 4-6.

7. Suwijn SR (2015) The diagnostic accuracy of dopamine transporter SPECT imaging to detect nigrostriatal cell loss in patients with Parkinson's disease or clinically uncertain parkinsonism: a systematic review. EJNMMI Res 5: 2-9.

8. Mahlknecht P (2012) Is transcranial sonography useful to distinguish drug-induced parkinsonism from Parkinson's disease? Mov Disord 27: 1194-1196.

9. Bouwmans AEP (2010) Transcranial sonography for the discrimination of idiopathic Parkinson's disease from atypical parkinsonian syndromes. Movement Disorder 10: 122-147.

10. Budisic M (2009) Distinguishing Parkinson's disease and essential tremor with transcranial sonography. Acta Neurol Scand 11: 917-921.

11. Sheng YJ (2012) Predictive value of transcranial sonography in the diagnosis of Parkinson disease. Chin J Med Imaging Technol 28: 1069-1071.

12. Wang XP, Peng J (2017) The application of transcranial ultrasound in early diagnosis of Parkinson's disease. Modern instruments and medicine 23: 7-9.

13. Hughes AJ (1992) Accuracy of clinical diagnosis of idiopathic Parkinson's disease: a clinico-pathological study of 100 cases. J Neurol Neurosurg Psychiatry 55: 181-184.

14. Parkinson's disease and dyskinesia group, C.s.o.n., Diagnostic criteria for Parkinson's disease in China. Chin J Neuro 49: 268-271.

15. Bowden J (2011) Quantifying, displaying and accounting for heterogeneity in the metaanalysis of RCTs using standard and generalised Q statistics. BMC Med Res Methodol 11: 41 .

16. Higgins JP (2003) Measuring inconsistency in meta-analyses. BMJ 327: 557-560.

17. Ioannidis JP, Patsopoulos NA, Evangelou E (2007) Uncertainty in heterogeneity estimates in meta-analyses. BMJ 335: 914-916.

18. Egger M (1997) Bias in meta-analysis detected by a simple, graphical test. BMJ 315 629-34.

19. Saltelli A (2002) Sensitivity analysis for importance assessment. Risk Anal 22: 579-590

20. Egger M (1997) Bias in meta-analysis detected by a simple, graphical test. BMJ Open 315: 629-634.

21. Wang R (2010) Significance of transcramal sonography in diagnosis of Parkinson's disease. China Journal of Modem Medicine 21: 822-825. 
22. Zhang YC, Fang JC, Sheng YJ (2010) Application of transcranial ultrasonography in diagnosis of Parkinson disease. Chin J Med Imaging Technol 26: 2255-2257.

23. Feng AZ (2012) The diagnostic accuracy of substantia nigra hyper-echogenicity by transcranial sonography (TCS) for the diagnosis of Parkinson's disease. Chinese community physician 14: 263.

24. Yang LJ, Zhang JF, Li YC (2012) Substantia nigra ultrasonography findings in Parkinson'S disease. China. J Gerial r Heart Brain Vessel 14: 390-393.

25. Luo WF (2012) Transcranial sonography on Parkinson's disease and essential tremor in a Chinese population. Neurol Sci 33: 1005-1009.

26. Cao CJ, Yu SY, Zuo LJ (2013) A study on the relationship between the change of echogenicity in substantia nigra by transcranial sonography and clinical features in patients with Parkinson's disease Chin J Clinicians (Electronic Edition) 7: 6904-6912.

27. Chen J, Huang YN, Sun XR (2013) The performance of transcranial ultrasound in the early onset Parkinson's disease. J Apoplexy Nerv Dis 30: 211-214.

28. Li C, He W, Zhang HQ (2013) Diagnositic value of trascranial sonography in detection of Parkinson disease. Chin J Med Ultrasound 10: 461-464

29. Liu X, Wang J, Sun SG (2013) Preliminary Application of Transcranial Ultrasonography in Parkinson's Disease. Chinese J Ultrasound Med 29: 577-580.

30. Zhuang JJ, Zheng YH, Xu XW (2015) The ultra earIy diagnosis of parkinson's disease by the enhanced substantia nigra echo. Eur Rev Med Pharmacol Sci 19: 4621-4626.

31. Zhang ZH, Jiang S, Wang PQ (2015) Application of motor symptoms $\square$ olfactory test and transcranial ultrasonography in diagnosis of Parkinson's disease China. $J$ Geriatr Heart Brain Vtssel Dis 17: 622-624.

32. Hu HY, Han MF, Xiong HH (2016) Application of transcranial sonography in diagnosis and differential diagnosis and the relationships study between substantia Iligra echo and clinical features of Parkinson's disease. Chin. J Minim Invasive Neurosurg 21: 392-395.

33. Qiao YN, Xie S, Gu HW (2016) Findings and clinical evaluation in patients of Parkinsonl S disease by Transcranial sonography. J Apoplexy Nerv Dis 33: 226-229.
34. Xu HW (2017) Motor symptoms and the sense of smell and transcranial doppler ultrasound in the diagnosis of Parkinson's disease. Journal of China Prescription Drug 15: 116-116.

35. Yang J, Wei ZL (2017) Clinical study of transcranial sonography in the patients with multiple system atrophy and Parkinson 's disease. Chinese. Journal of Practical Nervous Diseases 20: 83-86.

36. Dong ZF, Zhang YC, Sheng YJ (2017) Transcranial sonography in patients with Parkinson disease and Alzheimer disease. Chin J Med Imaging Technol 33: 514-517.

37. Zhu JW, Zhao C, Zhang W (2018) Transcranial sonography in diagnosis of Parkinson's disease. Clinical Focus 33: 413-415.

38. Liu XJ, Zhang L, Zhang YF (2018) Echogenic alteration in the raphe nuclei measured by transcranial sonography in patients with Parkinson disease and depression. Medicine 97: 50 .

39. Zhang LL, Yang TT, Zhang L (2018) Diagnostic value of transcranial ultrasound and non-motor symptoms assessment for early Parkinson's disease. Chinese Journal of Practical Nervous Diseases 21: 1577-1582.

40. Chitsaz A (2013) Transcranial sonography on Parkinson's disease and essential tremor. J Res Med Sci 18: S28-31.

41. Stockner H (2012) Is Transcranial sonography useful to distinguish scans without evidence of dopaminergic deficit patients from Parkinson's disease? Mov Disord 27: $1182-1185$

42. Doepp F, Plotkin M (2007) Brain parenchyma sonography and 123I-FP-CIT SPECT in Parkinson's disease and essential tremor. Mov Disord 23: 405-410.

43. Hall JM (2017) Hyperechogenicity of the substantia nigra in Parkinson's disease: Insights from two brothers with markedly different disease durations. Case Rep Neurol Med.

44. Walter U (2007) Transcranial brain parenchyma sonography in movement disorders: state of the art. Ultrasound Med Biol 33: 15-25.

45. Becker G (1994) Reliability of transcranial colour coded real-time sonography in assessment of brain tumours: correlation of ultrasound, computed tomography and biopsy findings. Neuroradiology 36: 585-590.

Copyright: (C2019 Liu B. This is an open-access article distributed under the terms of the Creative Commons Attribution License, which permits unrestricted use, distribution, and reproduction in any medium, provided the original author and source are credited. 